This item was submitted to Loughborough's Research Repository by the author.

Items in Figshare are protected by copyright, with all rights reserved, unless otherwise indicated.

\title{
Complex resonances in the water-wave problem for a floating structure
}

PLEASE CITE THE PUBLISHED VERSION

PUBLISHER

(c) Cambridge University Press

LICENCE

CC BY-NC-ND 4.0

\section{REPOSITORY RECORD}

Mclver, P.. 2019. "Complex Resonances in the Water-wave Problem for a Floating Structure". figshare. https://hdl.handle.net/2134/2220. 


\title{
Complex resonances in the water-wave problem for a floating structure
}

\author{
By P. MCIVER \\ Department of Mathematical Sciences, Loughborough University, Loughborough, Leics LE11 3TU, UK
}

(Received 30 June 2004 and in revised form 17 December 2004)

This work is concerned with the linearized theory of water waves applied to the motion of a floating structure that restricts in some way the motion of a portion of the free surface (an example of such a structure is a floating torus). When a structure of this type is held fixed in incident monochromatic waves, or forced to move time harmonically with a prescribed velocity, the amplitude of the fluid motion will have local maxima at certain frequencies of the forcing. These resonances correspond to poles of the scattering and radiation potentials when extended to the complex frequency domain. It is shown in this work that, in general, the positions of these poles in the scattering and radiation potentials will not coincide with the positions of the poles that appear in the velocity potential for the coupled problem obtained when the structure is free to move. The poles of the potential for the coupled problem are associated with the solution for the structural velocities of the equation of motion. When physical quantities such as the amplitude of the fluid motion are examined as a function of (real) frequency, there will in general be a shift in the resonant frequencies in going from the radiation and scattering problems to the coupled problem. The magnitude of this shift depends on the geometry of the structure and how it is moored.

\section{Introduction}

Resonant motions are often observed when water waves interact with an offshore structure and this paper is concerned with two particular types of resonant motion that appear within the linearized theory. The first of these motions is called here a 'sloshing resonance' and is associated with structures that restrict the motion of a portion of the free surface. Examples of structural geometries of this type are a half-immersed circular torus (Newman 1977), an array of vertical cylinders (Evans \& Porter 1997), and a submerged horizontal circular disk (Martin \& Farina 1997). An incident monochromatic wave will excite fluid motions around the fixed structure and in this scattering problem a graph of the magnitude of the hydrodynamic force on the structure as a function of wave frequency will display local maxima at particular frequencies. Sloshing resonances may also be observed in the radiation problem when such structures are forced to oscillate and characteristic rapid changes are observed in the added mass and damping coefficients near a resonant frequency (in particular, the phenomenon of negative added mass may be observed). Throughout this paper the term 'sloshing resonance' will be reserved to describe a resonance displayed by a scattering or radiation potential.

When a frequency-domain potential is regarded as a function of complex frequency, a resonance corresponds to a pole in the complex-frequency domain that lies close to the real frequency axis. The position of the pole in the complex-frequency 
domain characterizes the resonance. The real part of the pole position gives the resonant frequency and the imaginary part determines the degree of damping in the corresponding fluid motion; the farther the pole is from the real frequency axis, the greater the damping. Such poles are often referred to as complex resonances, or scattering frequencies, and their relation to the behaviour of hydrodynamic coefficients has been investigated by a number of authors (e.g. Linton \& Evans 1992; Hazard \& Lenoir 1993; Evans \& Porter 1997; Martin \& Farina 1997).

For certain special geometries a pole corresponding to a sloshing resonance may be located on the real axis in the complex-frequency domain. Such geometries are so-called 'trapping structures' and at the resonant frequency the fixed structure can support a trapped mode which is a free oscillation of the fluid that has finite energy. McIver (1996b) constructed the first structure of this type, and since then many others have been discovered in both the two- and three-dimensional water-wave problems. The consequences of the existence of trapped modes for hydrodynamic coefficients have been studied by Newman (1999) and McIver (2003).

The second type of resonant motion of concern here is associated with the free motion of a structure in water and will be called a 'motion resonance'. (Here the term 'free motion' is used to mean that the structure is able to respond to the forces generated by the fluid motion and includes the case of motion excited by incident waves. This contrasts with the scattering and radiation problems in which the structure is either held fixed or forced to move in a prescribed fashion.) A motion resonance may arise when the equations of motion for the structure contain one or more terms that describe spring effects due to moorings or, in the case of the vertical motion of a surface-piercing structure, due to the hydrostatic restoring force. Such resonances are well known to offshore engineers and are discussed in many textbooks (e.g. Faltinsen 1990, p. 68). When the frequency-domain velocity of the structure is regarded as a function of complex frequency, such resonances are associated with poles of the velocity (and hence of the velocity potential for the fluid motion) that again lie close to the real frequency axis. There is no restriction on the structural geometry for it to display a motion resonance.

The subject of this work is the free motion of a structure that displays a sloshing resonance in the scattering and/or one or more of the radiation problems, and is moored and/or surface piercing so that both types of resonance discussed above are present. The theoretical description of the situation requires the solution of a coupled problem involving the motions of both the structure and the fluid. In the usual formulation (see, for example, Linton \& McIver 2001, §1.3), the frequency-domain scattering and radiation potentials are introduced and the fluid velocity potential in the coupled problem is written as a linear combination of these potentials. For each structural mode of motion the corresponding radiation potential in this combination is multiplied by the velocity of the structure in that mode. As noted above, the poles associated with sloshing resonances will in general appear in the scattering and radiation potentials and those associated with motion resonances in the velocities. It might therefore be assumed that the fluid motion in the coupled problem displays both sets of complex resonances. It will be shown here that this is not the case. In general a sloshing resonance appearing in the scattering and radiation potentials will be annulled, and hence in the neighbourhood of the sloshing resonant frequency the motion is dominated by any nearby motion resonance. When going from the scattering and radiation problems to the coupled problem, there will appear to be a shift in a resonant frequency and the magnitude of this shift will depend upon the geometry of the structure and how it is moored. 
For simplicity of exposition, details will be given for the case of a structure that is able to move in translational modes only. However, the main argument given to support the annulment of sloshing resonances (in §7.1) applies equally well to the case of a structure that is able to respond to the fluid motion in all translational and rotational modes of motion. There are exceptional cases; for example, a sloshing resonance associated only with a particular mode of motion will be preserved in the solution to the coupled problem if the structure is unable to move in the mode corresponding to that resonance.

The cancellation of sloshing resonances is not entirely surprising as different solution operators are associated with, on the one hand, the scattering and radiation problems and, on the other hand, with the coupled-motion problem for the fluid and structure. In general, different solution operators will have different pole structures. However, the elimination of the sloshing resonances in coupled problems does not appear to have been demonstrated explicitly before, or the situations under which it will, or will not, occur discussed. This is the purpose of the present work.

The plan of the paper is as follows. In $\S 2$ the initial-value problem for a coupled fluid-structure problem is stated and in $\S 3$ the link with the frequency domain is described and, in particular, it is indicated how poles in frequency-domain quantities are related to the long-time asymptotics of the motions. The full formulation of the frequency-domain problem is given in $\S 4$. Sloshing and motion resonances are described in detail in $\S 5$ and, in particular, numerical results are given to illustrate the effects of sloshing resonances in both the frequency and time domains. The next two sections of the paper show how, in the coupled problem, the sloshing resonances will be annulled so that only motion resonances are usually displayed (exceptions occur when a structure is constrained to move only in certain modes). Motion in the absence of incident waves is discussed in $\S 6$ and motion excited by incident waves in $\S 7$. Finally, in $\S 8$ the main results are summarized and discussed and numerical results are given to illustrate the annulment of sloshing resonances.

\section{The initial-value problem}

An inviscid and incompressible fluid with a free surface is contained within a horizontal layer of depth $h$ that is bounded below by a rigid bed and extends to infinity in all horizontal directions. Cartesian coordinates $(x, y, z)$ are chosen with $z$ measured vertically upwards and the origin in the mean free surface. The fluid layer contains a structure that, for simplicity of exposition, is constrained to move only in the two translational modes corresponding to the $x$ - and $z$-directions. A displacement in mode $\mu$ is denoted by $x_{\mu}(t), \mu=0,1$, where $\mu=0$ corresponds to the heave $(z)$ direction, $\mu=1$ to the surge $(x)$ direction, and $t$ is time. (This unconventional mode indexing is used to unify the notation used here in the two- and three-dimensional problems.) The wetted surface of the structure is denoted by $\Gamma$, a normal coordinate to $\Gamma$ directed out of the fluid is denoted by $n$, and $n_{\mu}$ is the $\mu$ component of the unit normal to $\Gamma$.

The fluid motion is assumed to be irrotational so that it may be described by a velocity potential $\Phi(\boldsymbol{x}, z, t), \boldsymbol{x}=(x, y)$, that satisfies

$$
\nabla^{2} \Phi=0
$$

within the fluid, the bed condition

$$
\frac{\partial \Phi}{\partial z}=0 \quad \text { on } \quad z=-h
$$


the linearized free-surface condition

$$
\frac{\partial^{2} \Phi}{\partial t^{2}}=-g \frac{\partial \Phi}{\partial z} \quad \text { on } \quad z=0
$$

where $g$ is the acceleration due to gravity,

$$
\frac{\partial \Phi}{\partial n}=\sum_{\mu} \dot{x}_{\mu}(t) n_{\mu} \quad \text { on } \quad \Gamma
$$

and for all time

$$
\nabla \Phi \rightarrow 0 \text { as } \quad|\boldsymbol{x}| \rightarrow \infty .
$$

The motion is subject to the initial conditions

$$
\Phi(\boldsymbol{x}, 0,0)=P_{1}(\boldsymbol{x}), \quad \frac{\partial \Phi}{\partial t}(\boldsymbol{x}, 0,0)=P_{2}(\boldsymbol{x}),
$$

where $P_{1}(\boldsymbol{x})$ and $P_{2}(\boldsymbol{x})$ correspond to a prescribed incident wave. It is assumed that this incident wave is initially localized within a region away from the structure so that at $t=0$ the fluid adjacent to the structure is at rest.

The structure is moored by an arrangement of linear springs and dampers so that the equation of motion of the structure for displacements in direction $\mu$ is

$$
M \ddot{x}_{\mu}(t)=-\rho \iint_{\Gamma} \frac{\partial \Phi}{\partial t}(\boldsymbol{x}, z, t) n_{\mu} \mathrm{d} S-\sum_{\nu}\left[c_{\mu \nu} x_{\nu}(t)+\gamma_{\mu \nu} \dot{x}_{v}(t)\right]+F_{\mu}(t)
$$

where

$$
c_{\mu \nu}=\rho g W \delta_{0 \mu} \delta_{0 v}+k_{\mu \nu} .
$$

The constants $k_{\mu \nu}$ and $\gamma_{\mu \nu}$ describe the characteristics of respectively the springs and dampers in the moorings. The first term in the expression for $c_{\mu \nu}$ corresponds to the hydrostatic restoring force and $W$ is the waterplane area of the structure (the Kronecker deltas appear because there is no such force for horizontal motions). By Archimedes' principle the mass $M$ of the structure is the fluid density $\rho$ times the submerged volume of the structure. The first term on the right-hand side of (7) is the hydrodynamic force arising from the fluid motion, and $F_{\mu}(t)$ is an applied force. In addition, for each $\mu$ the initial displacement $x_{\mu}(0)$ and velocity $\dot{x}_{\mu}(0)$ of the structure must be prescribed.

\section{Long-time asymptotics and complex resonances}

Information about the initial-value problem described in $\$ 2$ may be obtained using a Fourier transform in time. The transforms of the potential $\Phi(\boldsymbol{x}, z, t)$ and displacement $x_{\mu}(t)$ are respectively

$$
\phi(x, z, \omega)=\int_{0}^{\infty} \Phi(x, z, t) \mathrm{e}^{\mathrm{i} \omega t} \mathrm{~d} t
$$

and

$$
\xi_{\mu}(\omega)=\int_{0}^{\infty} x_{\mu}(t) \mathrm{e}^{\mathrm{i} \omega t} \mathrm{~d} t
$$

and the inversion formula for the potential is

$$
\Phi(\boldsymbol{x}, z, t)=\frac{1}{\pi} \operatorname{Re} f_{0}^{\infty} \phi(x, z, \omega) \mathrm{e}^{-\mathrm{i} \omega t} \mathrm{~d} \omega .
$$


The present work is concerned with complex resonances that correspond to poles of the frequency-domain potential $\phi(x, z, \omega)$ in the complex- $\omega$-plane (causality requires that there can be no such poles in $\operatorname{Im} \omega>0$ ). In the case of a pole on the real- $\omega$-axis the path of integration in (11) must pass above the pole (see McIver, McIver \& Zhang 2003, §3).

A simple pole of $\phi$ at $\omega=\omega_{\mathrm{r}}-\mathrm{i} \epsilon$, with $\omega_{\mathrm{r}}>0$ and $\epsilon \geqslant 0$, gives

$$
\phi(x, z, \omega) \sim \frac{\psi(x, z)}{\omega-\left(\omega_{\mathrm{r}}-\mathrm{i} \epsilon\right)} \quad \text { as } \quad \omega \rightarrow \omega_{\mathrm{r}}-\mathrm{i} \epsilon,
$$

and it then follows from (11) that, for a fixed point in space, as $t \rightarrow \infty$ the contribution from this pole gives

$$
\Phi(x, z, t) \sim-2 \operatorname{Re}\left\{\mathrm{i} \psi(\boldsymbol{x}, z) \mathrm{e}^{-\mathrm{i} \omega_{\mathrm{r}} t}\right\} \mathrm{e}^{-\epsilon t}
$$

(see Maskell \& Ursell 1970). In general this is a damped oscillation of the fluid, but for $\epsilon=0$ the oscillation persists for all time and this is known as a trapped mode. Each simple pole of $\phi$ will contribute a similar term to the large-time asymptotics of $\Phi$ and a corresponding result holds for the displacement of the structure. Although they will not be considered here, higher-order poles may occur (for example, the frequency-domain potential for the forced oscillations of a trapping structure has a double pole when the forcing frequency corresponds to the trapped-mode frequency; see McIver et al. 2003).

For a floating structure on deep water, in both two and three dimensions, the decay of a vertical motion is ultimately algebraic (Kotik \& Lurye 1964; Ursell 1964) and not a damped oscillation of the form given by (13). (In two dimensions the decay is like $t^{-2}$ and in three dimensions like $t^{-4}$.) For the two-dimensional problem, Ursell (1964) shows how the algebraic decay arises from a logarithmic branch point in the velocity potential at the origin in the complex-frequency domain. In practice, when such algebraic decay does occur it is likely that it will be discernible only after a very long time when the motion is very small (Kotik \& Lurye 1968; Maskell \& Ursell 1970).

The function $\psi(\boldsymbol{x}, z)$ that appears in (12) and (13) is an eigenfunction of the homogeneous problem in the complex frequency domain (see $\S 7.1$ below) and will, in general, grow as $|\boldsymbol{x}| \rightarrow \infty$ (Hazard \& Lenoir 1993, § 4.3). However, at any particular time, the fluid motion in the time-domain problem decays to zero as $|\boldsymbol{x}| \rightarrow \infty$. Consequently, at large distances from the structure the asymptotic behaviour described by (13) will become apparent only after a time that is sufficiently long for the decay in time to offset the growth of the eigenfunction. An explicit approximation to a potential that displays a complex resonance is given by Newman (1974) who considered the two-dimensional problem of wave scattering by two closely spaced vertical barriers. Sufficiently far from the gap between the barriers, the effect of the gap is approximated by a potential $m(\omega) G(x, z, \omega)$, where $G$ is a standard frequency-domain wave source (Green's function) and $m$ has a pole at a complex frequency $\omega_{e}$, say, with $\operatorname{Im} \omega_{e}<0$. In this case the eigenfunction

$$
\psi(x, z) \approx m_{e} G\left(x, z, \omega_{e}\right)
$$

where $m_{e}$ is the residue of $m(\omega)$ at $\omega=\omega_{e}$. As $|x| \rightarrow \infty, G$ behaves as an outgoing wave which for a complex frequency $\omega_{e}$ grows with increasing $|x|$. In general, for water of constant depth $h$, a two-dimensional eigenfunction will have the form

$$
\psi(x, z)=\sum_{m=0}^{\infty} A_{m} \mathrm{e}^{-k_{m}|x|} \cos k_{m}(y+h)
$$


for sufficiently large $|x|$. Here each $k_{m}$ is a solution of

$$
k_{m} \tan k_{m} h=-\omega_{e}^{2} / g
$$

and (Hazard \& Lenoir 1993, § B.5)

$$
\operatorname{Re} k_{0}<0, \quad \operatorname{Im} k_{0}<0 \text { and } \operatorname{Re} k_{m}>0, \quad \operatorname{Im} k_{m}>0 \text { for } m \geqslant 1,
$$

so that only the first term in (15) grows with increasing $|x|$. The rate of growth of the eigenfunction depends on the distance of the pole from the axis through the solution of (16).

\section{The equations of motion}

The frequency-domain potential $\phi$ is conventionally decomposed as (see, for example, Linton \& McIver 2001, §1.3)

$$
\phi(x, z, \omega)=\phi_{\mathrm{S}}(\boldsymbol{x}, z, \omega)+\sum_{\mu} v_{\mu}(\omega) \phi_{\mu}(\boldsymbol{x}, z, \omega),
$$

where the scattering potential $\phi_{\mathrm{S}}$ satisfies

$$
\frac{\partial \phi_{\mathrm{S}}}{\partial n}=0 \quad \text { on } \quad \Gamma
$$

each radiation potential $\phi_{\mu}$ satisfies

$$
\frac{\partial \phi_{\mu}}{\partial n}=n_{\mu} \quad \text { on } \quad \Gamma
$$

and, from the Fourier transform of (4),

$$
v_{\mu}(\omega)=-\mathrm{i} \omega \xi_{\mu}(\omega)-x_{\mu}(0) .
$$

Fourier transformation of the equation of motion (7) shows that these frequencydomain velocities satisfy

$$
\begin{aligned}
\sum_{\nu}\left\{c_{\mu \nu}-\mathrm{i} \omega \gamma_{\mu \nu}\right\} v_{\nu}(\omega)-\omega^{2} M v_{\mu}(\omega) \\
\quad=\omega^{2} \rho \iint_{\Gamma} \phi n_{\mu} \mathrm{d} S-\mathrm{i} \omega\left[f_{\mu}(\omega)+M \dot{x}_{\mu}(0)\right]-\sum_{\nu} c_{\mu \nu} x_{\nu}(0), \quad \mu=0,1,
\end{aligned}
$$

or, after the introduction of the decomposition (18),

$$
\begin{aligned}
& \sum_{\nu}\left\{c_{\mu \nu}-\omega^{2}\right. {\left.\left[q_{\mu \nu}(\omega)+\mathrm{i} \gamma_{\mu \nu} / \omega\right]\right\} v_{\nu}(\omega)-\omega^{2} M v_{\mu}(\omega) } \\
&=-\mathrm{i} \omega\left[X_{\mu}(\omega)+f_{\mu}(\omega)+M \dot{x}_{\mu}(0)\right]-\sum_{\nu} c_{\mu \nu} x_{\nu}(0), \quad \mu=0,1 .
\end{aligned}
$$

Here

$$
X_{\mu}(\omega)=\mathrm{i} \omega \rho \iint_{\Gamma} \phi_{\mathrm{S}}(\boldsymbol{x}, z, \omega) n_{\mu} \mathrm{d} S
$$

is the exciting force in direction $\mu$ corresponding to $\phi_{\mathrm{S}}, f(\omega)$ is the Fourier transform of $F(t)$, and the complex force coefficient

$$
q_{\mu \nu}(\omega)=\rho \iint_{\Gamma} \phi_{\nu}(\boldsymbol{x}, z, \omega) n_{\mu} \mathrm{d} S .
$$


For real $\omega$,

$$
q_{\mu \nu}(\omega)=a_{\mu \nu}(\omega)+\frac{\mathrm{i} b_{\mu \nu}(\omega)}{\omega}
$$

where $a_{\mu \nu}$ and $b_{\mu \nu}$ are respectively the real-valued added mass and damping coefficients.

The potential $\phi$ and the displacement $\xi_{\mu}$ can be defined in the upper half of the complex- $\omega$-plane through the Fourier transforms in (9) and (10) and, moreover, they will be analytic functions throughout $\operatorname{Im} \omega>0$. In general the asymptotic behaviours of $\Phi$ and $x_{\mu}$ for large time will prevent the definition of $\phi$ and $\xi_{\mu}$ throughout the lower half-plane using (9) and (10). However, analytic continuation into the lower half-plane is possible in a neighbourhood $\mathscr{W}$ of any segment of the real axis that is free of singularities. The definitions (24) and (25) also allow the exciting force $X_{\mu}$ and complex force coefficient $q_{\mu \nu}$ to be defined as analytic functions of $\omega$ throughout $\mathscr{W}$, and the uniqueness principle of complex-variable theory then implies that equations (22) and (23) also hold in $\mathscr{W}$.

\section{Sloshing and motion resonances}

Two types of complex resonance are discussed in this paper. The first, called here a sloshing resonance, is associated with a pole of the radiation potential $\phi_{\mu}$ and/or the scattering potential $\phi_{\mathrm{S}}$. When the incident wave is extracted, the boundary-value problem for the scattering potential $\phi_{\mathrm{S}}$ differs from that for $\phi_{\mu}$ only by the data specified on $\Gamma$, the solution operators are identical and as consequence the two potentials will have closely related pole structures. However, it is possible to have a pole in $\phi_{\mathrm{S}}$ and no corresponding pole in a $\phi_{\mu}$, and vice versa. The relation between the two pole structures is discussed further in $\S 7$. Here it will be assumed that all poles in $\phi_{\mathrm{S}}$ and $\phi_{\mu}$ are simple poles. As far as the author is aware, there is no evidence for the existence of higher-order poles in these potentials, although it cannot be ruled out.

If there is a simple pole in the radiation potential at $\omega=\omega_{e} \equiv \omega_{\mathrm{r}}-\mathrm{i} \epsilon$, so that

$$
\phi_{v}(\boldsymbol{x}, z, \omega)=\frac{\psi_{v}(\boldsymbol{x}, z)}{\omega-\omega_{e}}+O(1) \quad \text { as } \quad \omega \rightarrow \omega_{e},
$$

then from (25) there is a singularity in the complex force coefficient $q_{\mu \nu}$, corresponding to that in $\phi_{v}$, with

$$
q_{\mu \nu}(\omega)=\frac{q_{\mu \nu}^{(-1)}}{\omega-\omega_{e}}+O(1) \quad \text { as } \quad \omega \rightarrow \omega_{e}
$$

unless the forcing is orthogonal to $\psi_{v}$ so that

$$
\iint_{\Gamma} \psi_{\nu}(\boldsymbol{x}, z) n_{\mu} \mathrm{d} S=0 .
$$

Here $q_{\mu \nu}^{(-1)}$ is independent of $\omega$. In the limit $\epsilon \rightarrow 0$ at least, $q_{\mu \mu}^{(-1)}$ must be real and negative to ensure that the corresponding damping coefficient is non-negative (Newman 1999; McIver 2003) and hence $\psi_{\mu}$ must also be real as $\epsilon \rightarrow 0$. Similarly, a pole in the scattering potential $\phi_{\mathrm{S}}$ will in general give corresponding poles in the exciting forces defined through (24).

The occurrence of sloshing resonances is illustrated numerically in figures 1-5 for a two-dimensional geometry consisting of a pair of closely spaced half-immersed circular cylinders each of radius $0.28 h$ and with centres at $x= \pm 0.31 h$ (the forced vertical 


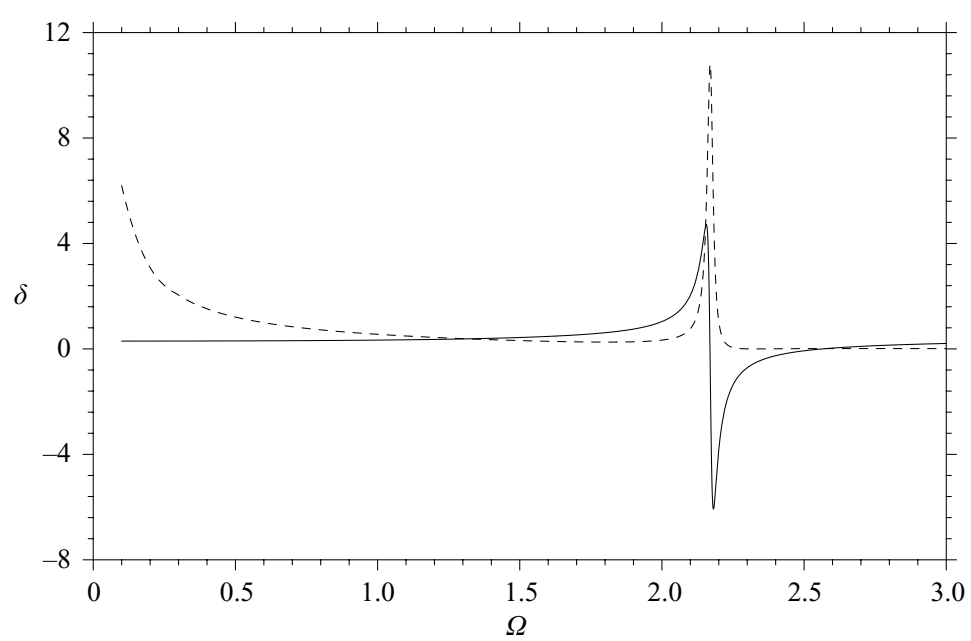

FIGURE 1. Non-dimensional hydrodynamic coefficients for two heaving half-immersed cylinders: added mass $a_{00} /\left(\rho h^{2}\right)(-)$ and damping $b_{00} /\left(\rho \omega h^{2}\right)(---)$.

oscillations of this structure and the estimation of the location of a pole are discussed by McIver et al. 2003). The time-domain results presented here were obtained by the method described by McIver et al. (2003) and the two-dimensional frequency-domain results by a standard boundary-integral code. Unless otherwise stated, in these results times are scaled by $T=\sqrt{h / g}$ and a non-dimensional frequency $\Omega=\omega \sqrt{h / g}$ is used. The heave added-mass and damping coefficients are shown in figure 1 and the rapid changes in these coefficients associated with a pole at $\Omega \approx 2.17-0.012 \mathrm{i}$ are clearly seen. The observed behaviours of the added mass and damping follow immediately from the real and imaginary parts of (28) provided that $q_{00}^{(-1)}$ is real and negative. There are additional sloshing resonances at higher frequencies but these are not shown.

When the structure described above is forced to oscillate at a specified frequency, then the fluid motion in the time domain reflects both the forcing and the sloshing resonances. The Fourier transform $v_{0}(\omega)$ of the forcing velocity has a pole on the real- $\omega$-axis at the forcing frequency and hence, from (18), there is corresponding pole in the velocity potential $\phi$ and so by (13) there is a persistent fluid oscillation at this frequency. In addition, decaying fluid oscillations will be excited at each frequency corresponding to a sloshing resonance. For a forcing frequency $\Omega=\pi / 2 \approx 1.57$, the free-surface motion midway between the cylinders is dominated by frequencies corresponding to the forcing frequency and the lowest sloshing resonance at $\Omega \approx 2.17$ leading to the 'beats' shown in figure 2. As $t \rightarrow \infty$ the fluid motion becomes a pure oscillation at the forcing frequency (the 'principle of limiting amplitude'). The discrete Fourier transform of the time signal given in figure 2 is shown in figure 3 and this confirms that the oscillation frequencies are indeed as argued above. Here $\left|u_{n}\right|$ is $N^{-1 / 2}$ times the amplitude of the Fourier component with index $n$, where $N$ is the number of samples in the signal, and the index $n$ is related to the non-dimensional frequency through

$$
\Omega=\frac{(n-1) 2 \pi T}{N \Delta t}
$$

where $\Delta t=0.005 T$ is the sampling interval. 


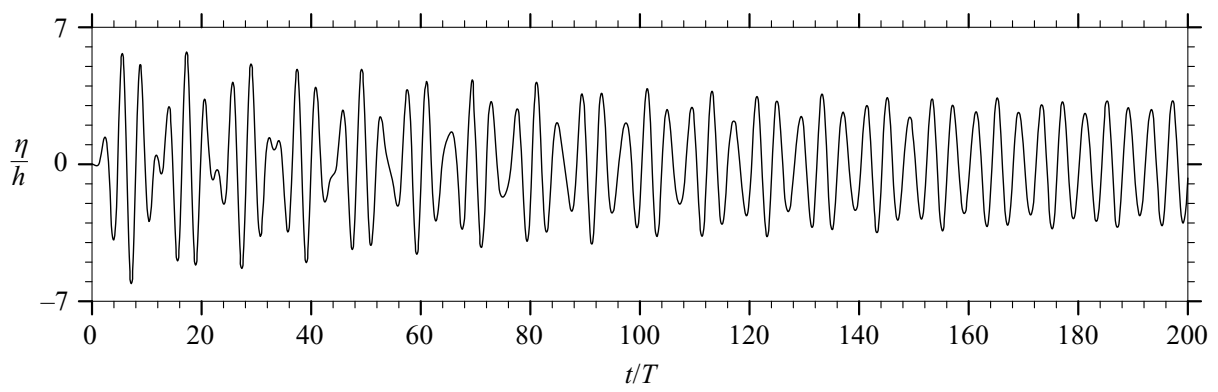

FIGURE 2. Two fixed half-immersed cylinders forced to oscillate at frequency $\Omega=\pi / 2$ : free-surface elevation $\eta$ at the mid-point of the internal free surface.

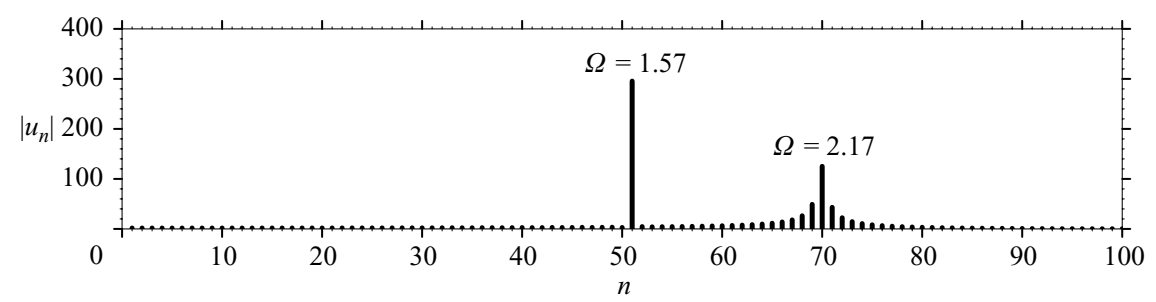

FIGURE 3. Discrete Fourier transform of the free-surface elevation given in figure 2.

In general, each pole in the radiation potential has a corresponding pole in the scattering potential (an exception is the case $\epsilon=0$ ) that leads, for example, to a pole in the frequency-domain exciting force and hence rapid changes in this force as the real frequency varies through values close to the pole location. In the time domain a pole in the scattering potential is associated with the excitation of fluid oscillations by wave incidence on a fixed structure and this is illustrated in figure 4 . The pair of fixed cylinders is subject to an incident Gaussian wave packet with surface elevation

$$
\eta(x, t)=\frac{1}{\sqrt{2 \pi} s} \int_{-\infty}^{\infty} \cos \left[k\left(x-x_{0}\right)-(k \tanh k h)^{1 / 2} t\right] \exp \left[-\left(k-k_{0}\right)^{2} /\left(2 s^{2}\right)\right] \mathrm{d} k .
$$

For the particular computations of figure 4 the standard deviation $s=1 / h$ and the mean wavenumber $k_{0}=4 / h$ (this corresponds to peak frequency $\Omega=2.00$ ); the offset $x_{0}$ is chosen so that initially there is negligible fluid motion in the vicinity of the structure. As the wave packet is scattered by the structure it excites a fluid oscillation between the cylinders and, as expected from (13), the oscillation dies away after the wave has passed. The discrete Fourier transform of the time signal given in figure 4 (extended to time $t=200 T$ ) is shown in figure 5 and this confirms that the oscillation frequency is consistent with the position of the pole in the frequency domain.

As demonstrated above, for $\epsilon \neq 0$, fluid oscillations in the time domain described by (13) can arise from forced oscillations of the structure or from wave incidence on the fixed structure. In the special case $\epsilon=0$, corresponding to a trapped mode of frequency $\omega_{\mathrm{r}}$, the situation is more complicated and the excitation of both steady and growing oscillations of the fluid by the forced oscillations of the structure is possible (McIver et al. 2003). However, a trapped mode cannot be excited by wave incidence on a fixed structure (McIver 1997) and hence there is no pole in the scattering potential $\phi_{\mathrm{S}}$ for $\epsilon=0$. 


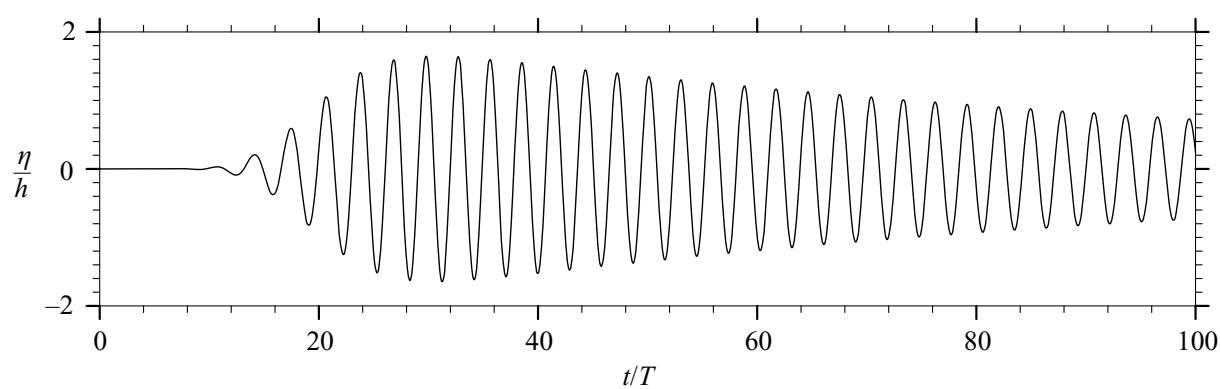

FIGURE 4. Two fixed half-immersed cylinders subject to an incident wave: free-surface elevation $\eta$ at the mid-point of the internal free surface.

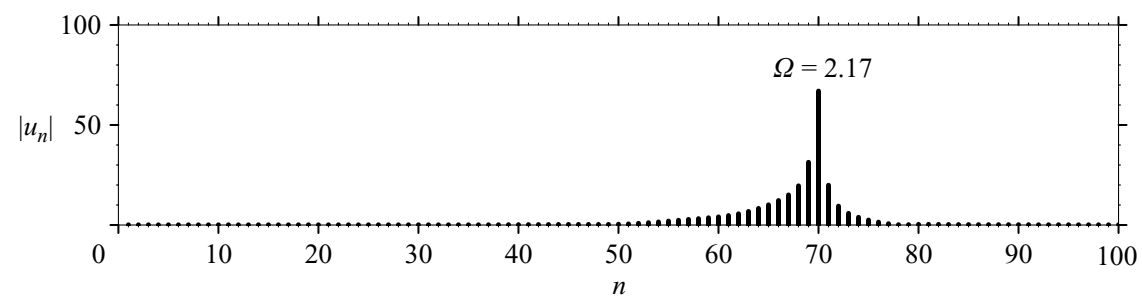

FIGURE 5. Discrete Fourier transform of the free-surface elevation given in figure 4.

The second complex resonance to be discussed, referred to here as a motion resonance, is associated with the motion of the structure and corresponds to a pole of the velocity $v_{\mu}$, for some $\mu$, given by the solution of (23). For the special case of motions constrained to mode $\mu$ only the location of a pole in the complex- $\omega$-plane is a solution of

$$
c_{\mu \mu}-\omega^{2}\left[M+q_{\mu \mu}(\omega)+\mathrm{i} \gamma_{\mu \mu} / \omega\right]=0 .
$$

For real $\omega$ the peak response is in the vicinity of a solution for $\omega$ of the real part of this equation provided that the pole is close to the real- $\omega$-axis. In the absence of a sloshing resonance (that is a pole in $q_{\mu \mu}$ ), there can only be a motion resonance for surge if the structure is moored, although heave resonance without moorings is possible because of the hydrostatic term in $c_{00}$. These types of resonance are well known to offshore engineers and a discussion is given, for example, by Faltinsen (1990, p. 68). In the presence of a sloshing resonance, a motion resonance (that is a solution of (32)) is possible in the absence of moorings for both heave and surge (Newman 1977, §6). The two types of resonance cannot occur at the same complex frequency.

Motion resonances may occur in the absence of the sloshing resonances that are a feature of structures that enclose a portion of the free surface. This enclosure may be complete, as in the numerical examples above, or partial, as for a twin-hulled vessel. For a floating structure that does enclose a portion of free surface both types of resonance can occur and there will be a mutual influence through the terms in (18). In the following two sections the interactions between the two types of resonance are examined in detail. In $\S 6$, the free motion of a structure in the absence of incident waves (so that $\phi_{\mathrm{S}}=0$ ) will be discussed, and this will be followed in $\S 7$ by an investigation of the motion excited by waves incident upon a structure that is free to move. 


\section{Motion in the absence of incident waves}

Here it is assumed that there is no applied force, so that $f_{\mu}=0$, and that there are no incident waves, so that the scattering potential $\phi_{\mathrm{S}}=0$ and the exciting force $X_{\mu}=0$. Motion in the time domain may be initiated, for example, by displacing the structure from its equilibrium position, allowing the fluid to settle, and then releasing the structure from rest. The dominant terms in the frequency-domain equations of motion (23) arise because of the poles in each $q_{\mu \nu}$ and this leads immediately to a set of homogeneous equations for the leading-order velocities. Excluding the pathological case when the coefficient matrix is singular, this gives

$$
v_{\mu}(\omega)=O\left(\omega-\omega_{e}\right) \quad \text { as } \quad \omega \rightarrow \omega_{e}
$$

and hence from (18) and (27)

$$
\phi(x, z, \omega)=\sum_{\mu} v_{\mu}(\omega) \phi_{\mu}(\boldsymbol{x}, z, \omega)=O(1) \quad \text { as } \quad \omega \rightarrow \omega_{e}
$$

so that is there is no pole in $\phi$. In other words, a sloshing resonance in $\phi_{\mu}$ is annulled by a corresponding zero in $v_{\mu}(\omega)$ and both the fluid and structural motions will be dominated by any motion resonances.

\section{Motion excited by incident waves}

In this section incident waves are included so that it is necessary to consider the full form (18) for the velocity potential. First of all an abstract argument is outlined to highlight the main results. This is followed by direct calculations (in both two and three dimensions) using various reciprocity relations that show directly how sloshing resonances that appear in the scattering and radiation potentials can be cancelled and hence not appear in the solution to a coupled problem.

\subsection{General formulation}

Here a discussion of sloshing and motion resonances is given in an abstract setting. Outline arguments without formal justification are given; for more detailed definitions and properties of some of the operators involved see Hazard \& Lenoir (1993).

In this subsection $\varphi$ denotes the frequency-domain potential with the incident wave extracted (thus $\phi=\varphi+\phi_{\mathrm{I}}$, where $\phi_{\mathrm{I}}$ is the potential of the incident wave). The boundary-value problem for $\varphi$ reduces to the boundary integral equation (Linton \& McIver 2001, §4.2)

$$
\frac{1}{2} \varphi(p ; \omega)=\iint_{\Gamma}\left(\varphi(q ; \omega) \frac{\partial G}{\partial n_{q}}(p, q ; \omega)-G(p, q ; \omega) g(q)\right) \mathrm{d} s_{q}, \quad p \in \Gamma,
$$

where $G(P, Q ; \omega)$ is the standard Green's function for the water-wave problem with field point $P$ and source point $Q$, lower-case letters denote points on $\Gamma$, and $g$ is the Neumann boundary data specified on $\Gamma$. For a structure moving in response to incident waves

$$
g=\boldsymbol{v} \cdot \boldsymbol{n}+g_{\mathrm{I}}
$$

where $\boldsymbol{v}=\left(v_{0}, v_{1}\right), \boldsymbol{n}=\left(n_{0}, n_{1}\right)$, and $g_{\mathrm{I}}=-\partial \phi_{\mathrm{I}} / \partial n$. With $\varphi(p ; \omega)$ determined for $p \in$ $\Gamma$, the solution for a point $P$ within the fluid domain is given by the integral representation

$$
\varphi(P ; \omega)=\iint_{\Gamma}\left(\varphi(q ; \omega) \frac{\partial G}{\partial n_{q}}(P, q ; \omega)-G(P, q ; \omega) g(q)\right) \mathrm{d} s_{q} .
$$


Equations (35) and (37) may be used to define the solution operator $\mathscr{R}_{\omega}$ that maps boundary data specified on the structural surface $\Gamma$ to the solution $\varphi$ (this operator is discussed in detail by Hazard \& Lenoir 1993, where it is denoted by $\mathscr{R}_{v}$ with $\left.v=\omega^{2} / g\right)$ so that

$$
\varphi=\mathscr{R}_{\omega}\left(\boldsymbol{v} \cdot \boldsymbol{n}+g_{\mathrm{I}}\right) .
$$

Note that the radiation potential $\phi_{\mu}=\mathscr{R}_{\omega} n_{\mu}$, and that $\mathscr{R}_{\omega} g_{\mathrm{I}}$ is the scattering potential $\phi_{\mathrm{S}}$ with the incident wave extracted.

For a surface-piercing structure, the integral equation (35) is not uniquely solvable for so-called irregular values of $\omega$ that correspond to non-trivial solutions of an interior Dirichlet problem (see Linton \& McIver 2001, §4.5). However, as this Dirichlet problem can be formulated in terms of a self-adjoint operator the irregular values of $\omega$ are necessarily real and hence do not lead to difficulties in the extension of $\mathscr{R}_{\omega}$ into the lower half of the complex- $\omega$-plane that is described below. There are alternative integral-equation formulations that are free of irregular values (see, for example, Lee, Newman \& Zhu 1996).

The extension to complex $\omega$ of the operator $\mathscr{R}_{\omega}$ follows from the evaluation of the Green's function at complex frequencies. If for some boundary data $g$ and complex frequency $\omega_{e}$

$$
\varphi=\mathscr{R}_{\omega} g \sim \frac{\psi_{e}}{\omega-\omega_{e}} \quad \text { as } \quad \omega \rightarrow \omega_{e},
$$

then examination of the limit $\omega \rightarrow \omega_{e}$ in (35) gives

$$
\frac{1}{2} \psi_{e}(p)=\iint_{\Gamma} \psi_{e}(q) \frac{\partial G}{\partial n_{q}}\left(p, q ; \omega_{e}\right) \mathrm{d} s_{q}, \quad p \in \Gamma .
$$

Thus sloshing resonances, that is the poles of the solution operator $\mathscr{R}_{\omega}$, are associated with the eigensolutions of the homogeneous boundary-value problem. Note that as all poles are assumed to be simple there is only a single eigenfunction associated with a complex resonance at $\omega=\omega_{e}$.

The equations of motion (22) may be rewritten as

$$
\boldsymbol{D} \boldsymbol{v}=\boldsymbol{P}\left(\varphi+\phi_{\mathrm{I}}\right)+\boldsymbol{u}
$$

where, for suitable $\varphi, \boldsymbol{P} \varphi$ has components

$$
[\boldsymbol{P} \varphi]_{\mu}=\omega^{2} \rho \iint_{\Gamma} \varphi n_{\mu} \mathrm{d} S, \quad \mu=0,1,
$$

the matrix $\boldsymbol{D}$ has components

$$
D_{\mu \nu}=c_{\mu \nu}-\mathrm{i} \omega \gamma_{\mu \nu}-\omega^{2} M \delta_{\mu \nu},
$$

and the vector $\boldsymbol{u}$ has components

$$
u_{\mu}=-\mathrm{i} \omega\left[f_{\mu}(\omega)+M \dot{x}_{\mu}(0)\right]-\sum_{\nu} c_{\mu \nu} x_{\nu}(0) .
$$

The alternative form (23) of the equations of motion may be written as

$$
\left(\boldsymbol{D}-\omega^{2} \boldsymbol{Q}\right) \boldsymbol{v}=-\mathrm{i} \omega \boldsymbol{X}+\boldsymbol{u}
$$

where $\boldsymbol{Q}$ is the matrix whose components are the complex force coefficients $q_{\mu \nu}(\omega)$, and $\boldsymbol{X}$ is the vector whose components are the exciting forces $X_{\mu}(\omega)$. Solutions of 
(45) for $v$ do not exist for those complex frequencies $\omega$ that are solutions of

$$
\operatorname{det}\left(\boldsymbol{D}-\omega^{2} \boldsymbol{Q}\right)=0
$$

and such frequencies are the motion resonances for the problem.

Elimination of $\boldsymbol{v}$ from (38) and (41) gives

$$
\mathscr{B}_{\omega} \varphi \equiv \varphi-\mathscr{R}_{\omega}\left(\boldsymbol{n} \cdot\left(\boldsymbol{D}^{-1} \boldsymbol{P} \varphi\right)\right)=\mathscr{R}_{\omega}\left(\boldsymbol{n} \cdot\left(\boldsymbol{D}^{-1}\left(\boldsymbol{P} \phi_{\mathrm{I}}+\boldsymbol{u}\right)\right)+g_{\mathrm{I}}\right) .
$$

It is straightforward to verify by substitution that $\varphi=\sum_{\mu} v_{\mu} \phi_{\mu}$ is a solution of

$$
\mathscr{B}_{\omega} \varphi=0 \text {, }
$$

and hence an eigenfunction of $\mathscr{B}_{\omega}$, provided that $\boldsymbol{v}$ is a non-trivial solution of

$$
\left(\boldsymbol{D}-\omega^{2} \boldsymbol{Q}\right) \boldsymbol{v}=\mathbf{0}
$$

and $\omega$ is a solution of (46). Thus any poles in $v$ will also appear in the solution to the coupled problem (47), and the corresponding eigenfunction is a coupled motion of the fluid and structure.

As noted above, sloshing resonances are associated with the poles of the operator $\mathscr{R}_{\omega}$ that appears on both sides of (47). The main aim here is to ascertain whether or not a sloshing resonance in one or more of the scattering and radiation potentials is preserved in the solution $\varphi$ to the coupled problem. In the remainder of this section it will be assumed that there is no forcing, so that there are no poles in $\boldsymbol{u}$, and that $\boldsymbol{D}^{-1}$ exists for any frequency under consideration (it is possible that $\operatorname{det} \boldsymbol{D}=0$ at a given complex frequency $\omega$ as long as the mooring characteristics have 'special' values).

Consideration is now given to $\mathscr{B}_{\omega} \varphi=\mathscr{R}_{\omega} g$ under the assumption that $\mathscr{R}_{\omega} g$ has a simple pole at $\omega=\omega_{e}$ that is associated with $\mathscr{R}_{\omega} g_{\text {I }}$ and/or at least one of the $\mathscr{R}_{\omega} n_{\mu}$. Consistency in equation (47) is obtained if there is a simple pole in $\mathscr{B}_{\omega} \varphi$ at $\omega=\omega_{e}$ that might arise from $\varphi$ directly and/or from $\mathscr{R}_{\omega}\left(\boldsymbol{n} \cdot\left(\boldsymbol{D}^{-1} \boldsymbol{P} \varphi\right)\right)$. As noted above, a pole in any potential at $\omega=\omega_{e}$ corresponds to the existence of an eigenfunction $\psi_{e}$. If a radiation potential $\phi_{\mu}$ exists at $\omega=\omega_{e}$, then an application of Green's theorem to $\phi_{\mu}$ and $\psi_{e}$ yields $\left[\boldsymbol{P} \psi_{e}\right]_{\mu}=0$ (in other words the last condition is necessary for the existence of $\phi_{\mu}$ ).

The following three cases cover all combinations of poles that are consistent with the assumptions given above.

(a) If the potential $\varphi$ and at least one of the radiation potentials $\phi_{\mu}=\mathscr{R}_{\omega} n_{\mu}$ have a pole at $\omega=\omega_{e}$ then, in general, there is a double pole in $\mathscr{B}_{\omega} \varphi$ arising from $\mathscr{R}_{\omega}\left(\boldsymbol{n} \cdot\left(\boldsymbol{D}^{-1} \boldsymbol{P} \psi_{e} /\left(\omega-\omega_{e}\right)\right)\right)$ and this is inconsistent with the initial assumption of a simple pole in $\mathscr{R}_{\omega} g$. However, if

$$
\boldsymbol{n} \cdot \boldsymbol{D}^{-1} \boldsymbol{P} \psi_{e}=0
$$

then $\mathscr{B}_{\omega} \varphi$ has only a simple pole and the combination of poles provides consistency. The normal vector $\boldsymbol{n}$ varies around the structure while, at a particular frequency, $\boldsymbol{D}^{-1} \boldsymbol{P} \psi_{e}$ is constant and hence (50) can be satisfied only if $\boldsymbol{D}^{-1} \boldsymbol{P} \psi_{e}=\mathbf{0}$. For a case in which $\phi_{v}$ exists, so that $\left[\boldsymbol{P} \psi_{e}\right]_{v}=0$, consistency can be obtained by choosing the mooring characteristics to ensure that $\left[\boldsymbol{D}^{-1} \boldsymbol{P} \psi_{e}\right]_{\mu}=0, \mu \neq \nu$. When consistency is obtained the scattering potential $\phi_{\mathrm{S}}$ may or may not have a pole.

For $\boldsymbol{P} \psi_{e} \neq \mathbf{0}$, the condition $\boldsymbol{D}^{-1} \boldsymbol{P} \psi_{e}=\mathbf{0}$ can be satisfied if $\operatorname{det} \boldsymbol{D}^{-1}=0$ at $\omega=\omega_{e}$ and this can be achieved at all frequencies by letting the mooring stiffness and damping tend to infinity for one mode of motion. For example, letting $c_{11}$ and $\gamma_{11}$ tend to 
infinity in $\boldsymbol{D}^{-1}$ gives

$$
\boldsymbol{D}^{-1}=\left(\begin{array}{cc}
\left(c_{00}-\mathrm{i} \omega \gamma_{00}-\omega^{2} M\right)^{-1} & 0 \\
0 & 0
\end{array}\right)
$$

which has the effect of constraining the motion to mode 0 (clearly the equations of motion then need some rewriting as it no longer makes sense to use $\left.\boldsymbol{D}=\left(\boldsymbol{D}^{-1}\right)^{-1}\right)$. Thus, if there is a pole in the surge potential $\phi_{1}$, with $\phi_{1} \sim \psi_{e} /\left(\omega-\omega_{e}\right)$ as $\omega \rightarrow \omega_{e}$, and the heave potential $\phi_{0}$ exists, so that $\left[\boldsymbol{P} \psi_{e}\right]_{0}=0$, then this form for $\boldsymbol{D}^{-1}$ ensures that $\boldsymbol{D}^{-1} \boldsymbol{P} \psi_{e}=\mathbf{0}$. However, as the structure is constrained to move in heave, $v_{1}=0$ and the pole in $\phi_{1}$ will not influence the potential $\varphi$ for the coupled problem. In this situation there will be a pole at $\omega=\omega_{e}$ in the scattering potential $\phi_{\mathrm{S}}$.

(b) If the potential $\varphi$ has a simple pole at $\omega=\omega_{e}$ and both of the radiation potentials $\phi_{\mu}=\mathscr{R}_{\omega} n_{\mu}$ exist for $\omega=\omega_{e}$, then $\mathscr{B}_{\omega} \varphi$ has a simple pole at $\omega=\omega_{e}$ that arises directly from $\varphi$ and consistency is obtained (there is no pole arising from $\mathscr{R}_{\omega}\left(\boldsymbol{n} \cdot\left(\boldsymbol{D}^{-1} \boldsymbol{P} \psi_{e} /\left(\omega-\omega_{e}\right)\right)\right)$ as the existence of the radiation potentials implies $\left.\boldsymbol{P} \psi_{e}=\mathbf{0}\right)$. The pole in $\varphi$ arises from the scattering potential $\phi_{\mathrm{S}}$.

The existence of both radiation potentials means that the eigenfunction associated with the pole in $\varphi$ is orthogonal to the forcing imposed by heave and surge oscillations, but the forcing from the scattering potential must excite the eigenfunction. The author is not aware of a structural geometry which displays these characteristics.

(c) If, at $\omega=\omega_{e}, \varphi$ exists and at least one of the radiation potentials $\phi_{\mu}$ has a simple pole then $\mathscr{B}_{\omega} \varphi$ has a simple pole at $\omega=\omega_{e}$ arising from $\mathscr{R}_{\omega}\left(\boldsymbol{n} \cdot\left(\boldsymbol{D}^{-1} \boldsymbol{P} \varphi\right)\right)$ and consistency is obtained. In this situation the scattering potential $\phi_{\mathrm{S}}$ may or may not have a pole. (It is assumed here that $\boldsymbol{D}^{-1} \boldsymbol{P} \varphi \neq \mathbf{0}$, that is the potential $\varphi$ induces a non-zero structural velocity. If $\varphi$ exists and $\boldsymbol{D}^{-1} \boldsymbol{P} \varphi=\mathbf{0}$ at $\omega=\omega_{e}$, then equation (47) yields consistency only if each of the scattering and radiation potentials has no pole at $\omega=\omega_{e}$.)

Thus, a pole in the radiation potential for a mode in which the structure is free to move gives no corresponding pole in the potential for the coupled problem. (This includes free motion constrained to a single mode and is in contrast to the example in case $(a)$ above where the pole occurs in the radiation potential for the mode in which there is no motion.) When there are corresponding poles in the scattering and radiation potentials there is cancellation in the coupled potential $\varphi$; explicit demonstrations of this cancellation are given in $\S \S 7.2$ and 7.3.

The cases discussed under items $(a)$ and $(b)$ above in which consistency is obtained mostly require very special conditions. The exception is that noted in $(a)$ in which the motion is constrained to be in a single mode. The 'usual' case is that described under item $(c)$. Whenever consistency is obtained, it follows from either (41) or (45) that the velocity $\boldsymbol{v}$ has no pole at $\omega=\omega_{e}$. Another apparent possibility, namely that $\varphi$ and all of the radiation potentials exist at $\omega=\omega_{e}$, so that the pole in $\mathscr{R}_{\omega} g$ arises from $\mathscr{R}_{\omega} g_{\mathrm{I}}$, gives no pole in $\mathscr{B}_{\omega} \varphi$ and this is inconsistent with the initial assumptions.

It should be noted that the form of equation (47) is unaltered when the structure is free to move in all translational and rotational modes. Thus, in particular, the situation described above in item $(c)$ will hold and sloshing resonances will not appear in the potential for the coupled problem.

\subsection{Two dimensions}

Attention is now turned to explicit calculations, based on reciprocal relations, for case $(c)$ of $\S 7.1$ in which one or both of the radiation potentials has a pole and the special circumstances mentioned under case $(a)$ do not arise. Consideration is 
first given to two-dimensional situations for which the $y$-coordinate is suppressed. In the frequency-domain scattering problem, a wave of amplitude $A$ and frequency $\omega$ is incident from large negative $x$ with potential

$$
\phi_{\mathrm{I}}=\frac{-\mathrm{i} g A}{\omega \cosh k h} \mathrm{e}^{\mathrm{i} k x} \cosh k(z+h)
$$

where the wavenumber $k$ is the solution of the dispersion relation

$$
\omega^{2}=g k \tanh k h .
$$

The scattering potential $\phi_{\mathrm{S}}(x, z, \omega)$ contains this incident wave as well as the wave field generated by scattering from the fixed structure. The radiation potentials $\phi_{\mu}(x, z, \omega)$, $\mu=0,1$, corresponding to forced oscillations of the structure with frequency $\omega$ have the asymptotic form

$$
\phi_{\mu} \sim A_{\mu}^{ \pm}(\omega) \mathrm{e}^{ \pm \mathrm{i} k x} \frac{\cosh k(z+h)}{\cosh k h} \text { as } x \rightarrow \pm \infty
$$

and it will be assumed that the complex amplitudes $A_{\mu}^{ \pm}$inherit the pole structure of $\phi_{\mu}$ so that

$$
A_{\mu}^{ \pm}(\omega) \sim \frac{\alpha_{\mu}^{ \pm}}{\omega-\omega_{e}} \quad \text { as } \quad \omega \rightarrow \omega_{e},
$$

where $\alpha_{\mu}^{ \pm}$are complex constants (there is substantial numerical evidence to support this assumption because of the link between the far-field waves and the damping coefficient). McIver (1996a) shows that the scattering potential can be expressed in terms of the radiation potentials as

$$
\phi_{\mathrm{S}}=\frac{-\mathrm{i} g A}{\omega \bar{D}}\left[\overline{A_{1}^{+}}\left(\phi_{0}-\bar{\phi}_{0}\right)-\overline{A_{0}^{+}}\left(\phi_{1}-\bar{\phi}_{1}\right)\right]
$$

where

$$
D=A_{0}^{+} A_{1}^{-}-A_{0}^{-} A_{1}^{+}
$$

(note that McIver uses a different scaling for the scattering potential and a different numbering system for the oscillation modes).

Each radiation potential $\phi_{\mu}$ can be expressed as the Fourier transform of a timedomain potential (cf. equation (9)) and it follows that for real frequencies $\overline{\phi_{\mu}}(x, z, \omega)=$ $\phi_{\mu}(x, z,-\omega)$ and this relation can then be used to extend $\overline{\phi_{\mu}}(x, z, \omega)$, and hence $\overline{A_{\mu}^{ \pm}}(\omega)$, into the lower half of the complex- $\omega$-plane. As $\phi_{\mu}(x, z, \omega)$ is analytic for $\operatorname{Im} \omega>0$, $\overline{\phi_{\mu}}(x, z, \omega)$ and $\overline{A_{\mu}^{ \pm}}(\omega)$ are analytic for $\operatorname{Im} \omega<0$. Thus, all quantities in equation (56) are analytic functions of $\omega$ in any neighbourhood $\mathscr{W}$ of the real frequency axis for which $\phi_{\mu}$ is free of singularities and hence (56) also holds throughout $\mathscr{W}$.

Consider the case when there is a pole in both radiation potentials at $\omega=\omega_{e}$ (a pole in only one radiation potential can be treated by a simplified argument). From established reciprocity relations for the water-wave problem (see, for example, Chapter 1 of Linton \& McIver 2001) the exciting force

$$
X_{\mu}(\omega)=-2 \mathrm{i} \omega \rho c_{g} A A_{\mu}^{-}(\omega) \sim \frac{-2 \mathrm{i} \omega_{e} \rho c_{g} A \alpha_{\mu}^{-}}{\omega-\omega_{e}} \quad \text { as } \quad \omega \rightarrow \omega_{e}
$$

and, for real frequencies, the damping coefficient

$$
b_{\mu \nu}(\omega)=\frac{\rho \omega^{2} c_{g}}{g}\left(A_{\mu}^{-}(\omega) \overline{A_{\nu}^{-}}(\omega)+A_{\mu}^{+}(\omega) \overline{A_{\nu}^{+}}(\omega)\right) .
$$


Here $c_{g}$ is the group velocity which is a function of frequency. In the limit $\omega \rightarrow \omega_{e}, c_{g}$ is evaluated at $\omega=\omega_{e}$ although this will not be stated explicitly. For real frequencies

$$
b_{\mu \nu}(\omega)=\frac{\omega}{2 \mathrm{i}}\left(q_{\mu \nu}(\omega)-\overline{q_{\mu \nu}}(\omega)\right)
$$

and this can be used to extend (59) into the lower half of the complex- $\omega$-plane in a similar way to that described above for equation (56). As $\omega \rightarrow \omega_{e}$ the behaviour of $q_{\mu \nu}$ is given by (28) and $\overline{q_{\mu \nu}}$ is analytic and so, after equating the residues at the pole, equation (59) yields

$$
q_{\mu \nu}^{(-1)}=\frac{2 \mathrm{i} \rho w_{e} c_{g}}{g}\left(\alpha_{\mu}^{-} \overline{A_{\nu}^{-}}\left(\omega_{e}\right)+\alpha_{\mu}^{+} \overline{A_{\nu}^{+}}\left(\omega_{e}\right)\right) .
$$

As $\omega \rightarrow \omega_{e}$, the dominant terms in the equations of motion (23) arise from the poles in the exciting forces $X_{\mu}$ and the complex force coefficients $q_{\mu v}$, and a simplified set of equations for the $v_{\mu}$ is readily obtained. Under the assumption that the coefficient matrix is non-singular, solution of these equations and substitution from above gives

$$
v_{0} \sim \frac{\mathrm{i} g A}{\omega_{e}} \frac{\overline{A_{1}^{+}}\left(\omega_{e}\right)}{\bar{D}\left(\omega_{e}\right)} \quad \text { and } \quad v_{1} \sim-\frac{\mathrm{i} g A}{\omega_{e}} \frac{\overline{A_{0}^{+}}\left(\omega_{e}\right)}{\bar{D}\left(\omega_{e}\right)} \quad \text { as } \quad \omega \rightarrow \omega_{e} .
$$

From the pole structures in equations (27) and (55) for the radiation potentials and their far-field forms, (56) yields

$$
\phi_{\mathrm{S}}(x, z, \omega) \sim \frac{-\mathrm{i} g A}{\omega_{e} \bar{D}\left(\omega_{e}\right)}\left(\frac{\overline{A_{1}^{+}}\left(\omega_{e}\right) \psi_{0}(x, z)-\overline{A_{0}^{+}}\left(\omega_{e}\right) \psi_{1}(x, z)}{\omega-\omega_{e}}\right) \quad \text { as } \quad \omega \rightarrow \omega_{e}
$$

and it then follows immediately from (18) that the poles in $\phi_{\mathrm{S}}$ and the radiation potentials cancel and that the resonance is annulled.

\subsection{Three dimensions}

Here three-dimensional structures are considered, but with attention restricted to those structures that have a vertical axis of symmetry, and results similar to those given in $\S 7.2$ are obtained. For this situation it is convenient to employ cylindrical polar coordinates $(r, \theta, z)$ where $r$ and $\theta$ are related to $x$ and $y$ in the standard way. The incident wave (52) may be expressed in terms of polar coordinates using the identity

$$
\mathrm{e}^{\mathrm{i} k x}=\sum_{\mu=0}^{\infty} \varepsilon_{\mu} \mathrm{i}^{\mu} J_{\mu}(k r) \cos \mu \theta
$$

(Abramowitz \& Stegun 1964, §9.1) where $J_{\mu}$ is a Bessel function of order $\mu, \varepsilon_{0}=1$, and $\varepsilon_{\mu}=2$ for $\mu \geqslant 1$. It follows from the asymptotic form of the Bessel functions that $\phi_{\mathrm{I}} \sim \frac{-\mathrm{i} g A \cosh k(z+h)}{2 \omega \cosh k h}\left(\frac{2}{\pi k r}\right)^{1 / 2} \sum_{\mu=0}^{\infty} \varepsilon_{\mu}\left[\mathrm{e}^{\mathrm{i}(k r-\pi / 4)}+(-1)^{\mu} \mathrm{e}^{-\mathrm{i}(k r-\pi / 4)}\right] \cos \mu \theta \quad$ as $\quad r \rightarrow \infty$.

The last expression contains both incoming and outgoing waves; the incoming components are the only such waves that appear in the scattering potential $\phi_{\mathrm{S}}$.

To obtain the three-dimensional equivalent of the relation (56) between scattering and radiation potentials, it is necessary to introduce the sequence of radiation potentials $\phi_{\mu}, \mu=0,1,2, \ldots$, that satisfy

$$
\frac{\partial \phi_{\mu}}{\partial n}=N_{\mu} \quad \text { on } \quad \Gamma
$$


where $N_{\mu} \propto \cos \mu \theta$ and is real valued; specifically, $N_{0} \equiv n_{0}$ and $N_{1} \equiv n_{1}$ (explicit forms for $N_{\mu}, \mu \geqslant 2$, are not needed here). In the far field the radiated field satisfies

$$
\phi_{\mu} \sim A_{\mu}(\omega)\left(\frac{2}{\pi k r}\right)^{1 / 2} \frac{\mathrm{e}^{\mathrm{i} k r-\mathrm{i} \pi / 4} \cos \mu \theta \cosh k(z+h)}{\cosh k h} \quad \text { as } \quad r \rightarrow \infty .
$$

By construction the form

$$
\psi=\sum_{\mu=0}^{\infty} B_{\mu}\left(\phi_{\mu}-\overline{\phi_{\mu}}\right)
$$

has zero normal derivative on $\Gamma$ and

$$
\psi \sim \frac{\cosh k(z+h)}{\cosh k h}\left(\frac{2}{\pi k r}\right)^{1 / 2} \sum_{\mu=0}^{\infty} B_{\mu}\left[A_{\mu} \mathrm{e}^{\mathrm{i}(k r-\pi / 4)}-\overline{A_{\mu}} \mathrm{e}^{-\mathrm{i}(k r-\pi / 4)}\right] \cos \mu \theta \quad \text { as } \quad r \rightarrow \infty
$$

By matching the incoming waves in $\psi$ to those in $\phi_{\mathrm{S}}$, both of these potentials are forced to satisfy the same boundary value problem and hence, under the assumption that the solution for $\phi_{\mathrm{S}}$ is unique at the particular frequency,

$$
\phi_{\mathrm{S}}=\frac{\mathrm{i} g A}{2 \omega} \sum_{\mu=0}^{\infty} \frac{\varepsilon_{\mu}(-1)^{\mu}}{\overline{A_{\mu}}}\left(\phi_{\mu}-\overline{\phi_{\mu}}\right) .
$$

The argument now proceeds in a similar way to the two-dimensional case. Suppose that there is a pole in $\phi_{\mu}$ at $\omega=\omega_{e}$, where $\mu$ takes the value of either zero or one, and that there are no coincident poles in any other $\phi_{\mu}$ (this is a consequence of the assumed axisymmetry). Thus in the limit $\omega \rightarrow \omega_{e}$

$$
\phi_{\mu}(\boldsymbol{x}, z, \omega) \sim \frac{\psi_{\mu}(\boldsymbol{x}, z)}{\omega-\omega_{e}}
$$

and the far-field amplitude coefficient

$$
A_{\mu}(\omega) \sim \frac{\alpha_{\mu}}{\omega-\omega_{e}} .
$$

Equation (69) can be extended into the complex plane as described in $\S 7.2$ for equation (56); it then follows from (70) that

$$
\phi_{\mathrm{S}}(\boldsymbol{x}, z, \omega) \sim \frac{\mathrm{i} g A \varepsilon_{\mu}(-1)^{\mu}}{2 \omega_{e} \overline{A_{\mu}}\left(\omega_{e}\right)} \frac{\psi_{\mu}(\boldsymbol{x}, z)}{\left(\omega-\omega_{e}\right)} \quad \text { as } \quad \omega \rightarrow \omega_{e} .
$$

Reciprocity relations (Linton \& McIver 2001, Chapter 1) yield

$$
X_{\mu}(\omega)=\frac{-4 \mathrm{i} \omega \rho c_{g} A(-1)^{\mu} A_{\mu}(\omega)}{k} \sim \frac{-4 \mathrm{i} \omega \rho c_{g} A(-1)^{\mu} \alpha_{\mu}}{k\left(\omega-\omega_{e}\right)} \quad \text { as } \quad \omega \rightarrow \omega_{e}
$$

and, for real frequencies,

$$
b_{\mu \mu}(\omega)=\frac{4 \rho \omega^{2} c_{g} A_{\mu}(\omega) \overline{A_{\mu}}(\omega)}{g k \varepsilon_{\mu}} .
$$

This last result can be extended into the complex frequency plane by a similar argument to that following equation (60), and then comparison with (28) yields

$$
q_{\mu \mu}^{(-1)}=\frac{8 \mathrm{i} \rho \omega_{e} c_{g} \alpha_{\mu} \overline{A_{\mu}}\left(\omega_{e}\right)}{g k \varepsilon_{\mu}}
$$




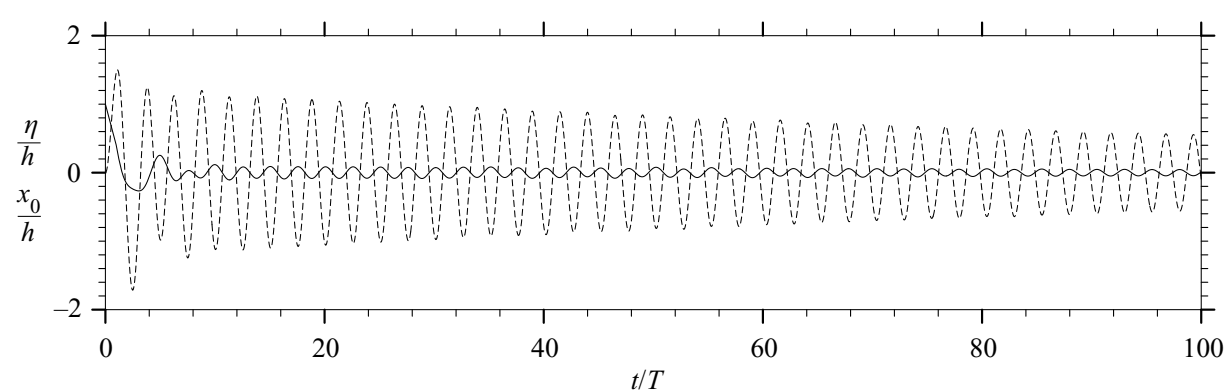

FIGURE 6. Release from rest of two freely floating half-immersed cylinders: free-surface elevation $\eta$ at the mid-point of the internal free surface $(---)$ and displacement $x_{0}$ of the structure (- $)$.

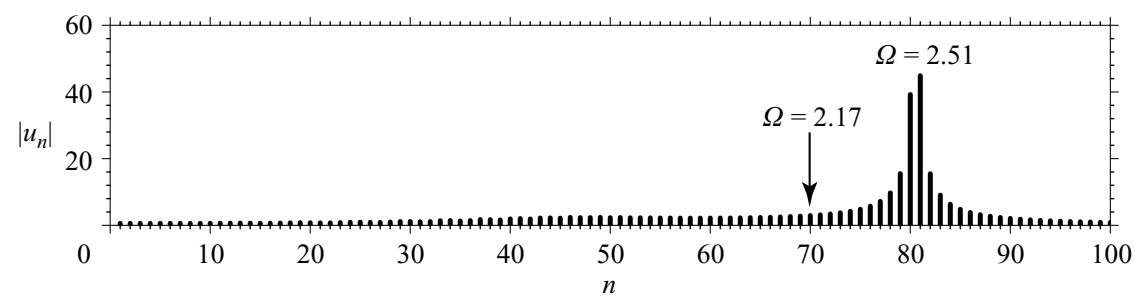

Figure 7. Discrete Fourier transform of the free-surface elevation given in figure 6.

Finally, because of the pole structure in the hydrodynamic coefficients, the dominant terms in the equation of motion give

$$
v_{\mu}(\omega) \sim \frac{g A(-1)^{\mu} \varepsilon_{\mu}}{2 \mathrm{i} \omega_{e} \overline{A_{\mu}}\left(\omega_{e}\right)} \quad \text { as } \quad \omega \rightarrow \omega_{e}
$$

and it is then a simple calculation to show from (18) that the pole in $\phi(x, z, \omega)$ is annulled.

\section{Discussion}

The decomposition of the fluid velocity potential into scattering and radiation potentials is commonly used in the description of the motion of a floating structure by the linearized theory of water waves. When the shape of the structure restricts the motion of a portion of the free surface, sloshing resonances appear in the scattering and radiation potentials. It has been demonstrated here that sloshing resonances do not, in general, appear in the solutions to coupled problems that arise when the structure is free to move. The solution will be dominated by motion resonances that arise from the components of the structural velocity. In practice, a motion resonance often occurs at a frequency close to each sloshing resonance so that the phenomenon described here is observed as a small shift of the resonance frequency when going from, say, a radiation problem to a coupled problem. Similar shifts in resonant frequency may be observed in simple mechanical systems.

In $\S 6$ a simple argument was used to show the absence of sloshing resonances for motion in the absence of incident waves. This result is illustrated here using numerical simulations in the timedomain for the same structure discussed in $\S 5$. The structure is constrained to move in heave and is not moored. As noted before there is a pole in the radiation potential corresponding to a sloshing resonance at $\Omega=2.17-0.012 \mathrm{i}$, 


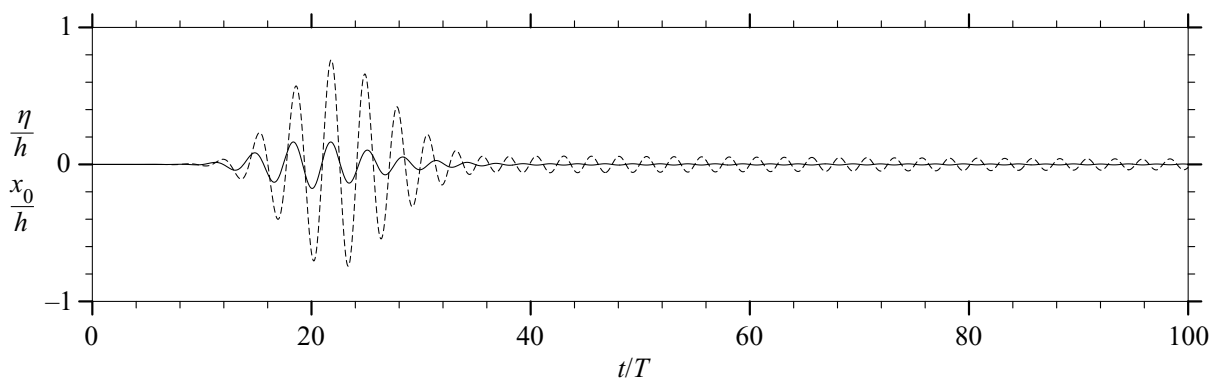

FiguRE 8 . Two freely floating half-immersed cylinders subject to an incident wave: free-surface elevation $\eta$ at the mid-point of the internal free surface $(---)$ and displacement $x_{0}$ of the structure $(-)$.

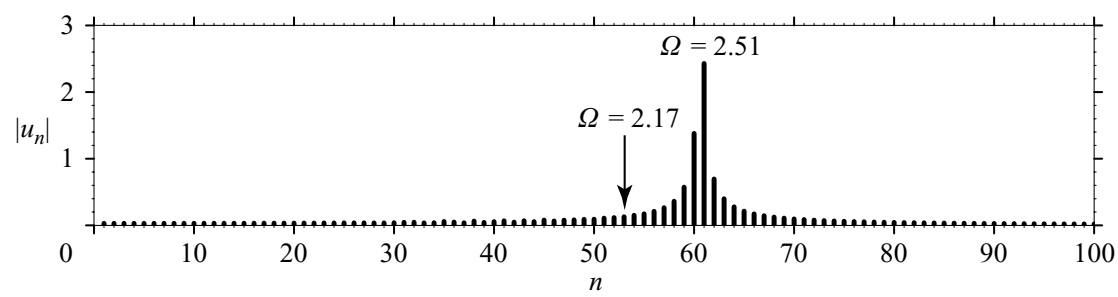

Figure 9. Discrete Fourier transform of the free-surface elevation given in figure 8.

while solution of the real part of (32) reveals that there is a motion resonance at about $\Omega=2.50$. Figures 6 and 7 correspond to the free motion of the structure that results from the initial conditions $x_{0}(0)=1, \dot{x}_{0}(0)=0$ so that the structure is displaced and released from rest. Figure 6 shows the free-surface elevation $\eta$ at the mid-point of the internal free surface and the structural displacement $x_{0}$ as functions of time. After about time $t=15$ both motions have settled to a decaying oscillation that appears to be dominated by a single frequency. This is confirmed by figure 7 which shows the discrete Fourier transform of the free-surface elevation shown in figure 6 (extended to time $t=$ $200 T$ ). The peak response is at the motion resonance while there is no discernible effect of the sloshing resonance, as is to be expected from the discussion above.

In $\S 7$, the annullment of sloshing resonances for a freely floating structure in incident waves was described and this is first illustrated in figures 8 and 9 with calculations in the time domain. The structure is again the two half-immersed cylinders described in $\S 5$, that exhibits a sloshing resonance for $\Omega \approx 2.17$, and the incident wave is that used for the results of figures 4 and 5. The structure is constrained to move in heave only and there are no moorings. Figure 8 shows the excitation of the free surface and of the structure caused by the passage of the wave packet, followed by decaying oscillations of a single frequency. The Fourier transform of the motion shown in figure 9 shows that the oscillation is at the frequency $\Omega \approx 2.51$ of the motion resonance and that there is no discernible effect of the sloshing resonance (to better reveal the motion obtained after the passage of the wave packet, figure 9 uses the time signal for $50 \leqslant t / T \leqslant 200$ ).

Complex resonances in the infinite-depth three-dimensional problem are illustrated in figure 10 for a half-immersed circular torus with free-surface intersection given by an annulus of outer radius $d$ and inner radius $0.2 d$. The calculations are in the frequency domain and were performed with version 5.4 of the radiation-diffraction panel code WAMIT. In figure $10(a)$ the submerged surface of the torus is modelled 

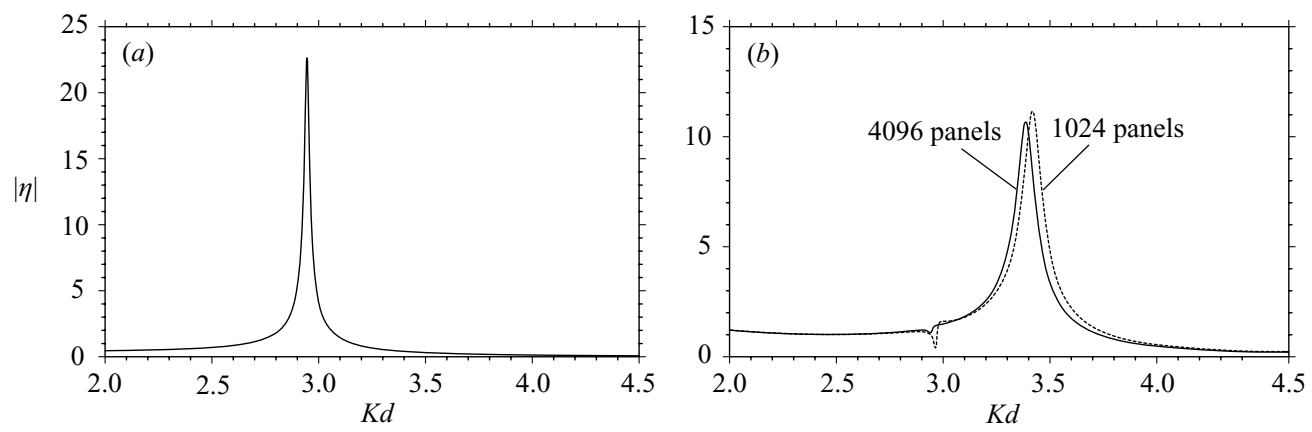

FIgURE 10. Half-immersed torus: amplitude $|\eta|$ of the free-surface elevation at the centre of the internal free surface $v$ s. frequency parameter $K d$ for $(a)$ a fixed torus, and $(b)$ a freely-floating torus.

by 4096 panels and in figure 10(b) further results are shown for 1024 panels (in both cases, additional panels within the waterplane area were used to remove irregular frequencies). Each part of the figure shows the magnitude of the free-surface elevation at the centre of the internal free surface as a function of the frequency parameter $K=\omega^{2} / g$. In figure $10(a)$ the torus is held fixed in incident waves and the spike for $K d \approx 2.94$ arises from a pole in the scattering potential $\phi_{\mathrm{S}}$. In figure $10(b)$ the torus is free to respond in heave to the incident waves so that there are contributions to the motion from both the scattering potential and the radiation potential. There is now a motion resonance at $K d \approx 3.39$, but some effects of the sloshing resonance remain because numerical errors prevent perfect cancellation between the contributions from the scattering and radiation problems. As the discretization of the structure is refined the kink in the curve arising from the sloshing resonances is reduced.

For this toroidal geometry there is a sloshing resonance in both the surge radiation potential and the scattering potential at $K d \approx 9.82$. For the problem discussed in the previous paragraph for which the structure is constrained to move in heave, the resonance in the surge potential does not influence the coupled-motion problem as the surge component of the velocity is zero. However, for example, the horizontal component of the hydrodynamic force on the heaving structure displays a resonance at $K d \approx 9.82$ as a result of the resonance in the scattering potential.

The following interpretation of the above results was suggested by J. N. Newman (private communication). For a sloshing resonance whose complex frequency is a small distance $\epsilon$ from the real frequency axis, the exciting force and damping coefficient are large, respectively $O\left(\epsilon^{-1 / 2}\right)$ and $O\left(\epsilon^{-1}\right)$, but from the equation of motion the velocity of the structure is $O\left(\epsilon^{1 / 2}\right)$. Thus, because of the large damping, only small structural motions are excited despite the large exciting force due to the incident wave. However, there is a direct connection between large fluid motions and large hydrodynamic pressure forces on the structure. As the motion of the structure is small, there cannot be a large net hydrodynamic force on the structure and hence there can be no large fluid motions associated with a sloshing resonance when the structure is free to move.

Trapped modes correspond to the limiting case $\epsilon=0$ so that the pole in a radiation potential that corresponds to a resonance is located on the real- $\omega$-axis. The fixed structure is then able to support free oscillations of the fluid with finite energy. In this case it is known that the scattering potential $\phi_{\mathrm{S}}$ exists at any real frequency $\omega_{\mathrm{r}}$ corresponding to a pole in a radiation potential (McIver 1997). It follows that the 
exciting force also has no pole and an identical argument to that given in $\S 6$ then shows that each pole in a radiation problem is annulled by a corresponding zero in the velocity. Hence a trapped mode cannot be excited by any free motion of a floating structure with, or without, incident waves. This statement is not contradicted by the results on the excitation of trapped modes given by McIver et al. (2003) because the situations described there involve the prescription of a structural velocity $\dot{x}_{\mu}(t)$ that is equivalent to the application of an external force $F_{\mu}(t)$ whose Fourier transform $f_{\mu}(\omega)$ has a pole at the trapped-mode frequency $\omega=\omega_{\mathrm{r}}$. With such a pole in $f_{\mu}$, equation (23) gives $v_{\mu}(\omega)=O(1)$ as $\omega \rightarrow \omega_{\mathrm{r}}$ and consequently the pole in $\phi_{\mu}$ at $\omega=\omega_{\mathrm{r}}$ is not annulled and the trapped mode is excited.

The author is grateful to C. M. Linton, P. A. Martin, and M. McIver for their comments on a draft of this paper.

\section{REFERENCES}

Abramowitz, M. \& STegun, I. A. 1964 Handbook of Mathematical Functions. National Bureau of Standards.

Evans, D. V. \& Porter, R. 1997 Near-trapping of waves by circular arrays of vertical cylinders. Appl. Ocean Res. 19, 83-99.

Faltinsen, O. M. 1990 Sea Loads on Ships and Offshore Structures. Cambridge University Press.

HazARD, C. \& LenoIr, M. 1993 Determination of scattering frequencies for an elastic floating body. SIAM J. Math. Anal. 24, 1458-1514.

Kotik, J. \& Lurye, J. 1964 Some topics in the theory of coupled ship motions. Proc. 5th Symp. on Naval Hydrodynamics, Bergen, Norway, 10-12 September 1964, pp. 407-424. Office of Naval Research, Washington.

Kotik, J. \& Lurye, J. 1968 Heave oscillations of a floating cylinder or sphere. Schiffstechnik 15, $37-38$.

LeE, C.-H., Newman, J. N. \& ZhU, X. 1996 An extended boundary integral equation method for the removal of irregular frequency effects. Intl J. Numer. Meth. Fluids 23, 637-660.

Linton, C. M. \& Evans, D. V. 1992 The radiation and scattering of surface waves by a vertical cylinder in a channel. Phil. Trans. R. Soc. Lond. A 338, 325-357.

Linton, C. M. \& McIver, P. 2001 Handbook of Mathematical Techniques for Wave/Structure Interactions. Chapman \& Hall/CRC, Boca Raton.

Martin, P. A. \& FARINA, L. 1997 Radiation of water waves by a heaving submerged horizontal disc. J. Fluid Mech. 337, 365-379.

Maskell, S. J. \& Ursell, F. 1970 The transient motion of a floating body. J. Fluid Mech. 44, 303-313.

MCIvER, M. $1996 a$ Global relationships between two-dimensional water wave potentials. J. Fluid Mech. 312, 299-309.

MCIVER, M. $1996 b$ An example of non-uniqueness in the two-dimensional linear water wave problem. J. Fluid Mech. 315, 257-266.

McIver, M. 1997 Resonances in the unbounded water wave problem. Proc. 12th Intl Workshop on Water Waves and Floating Bodies, Carret-le-Rouet, France, 16-19 March 1997, pp. 177-180.

McIver, M. 2003 The influence of a trapped mode on a radiation potential. Proc. 18th Intl Workshop on Water Waves and Floating Bodies, Le Croisic, France, 6-9 April 2003.

McIver, P., McIver, M. \& Zhang, J. 2003 Excitation of trapped modes by the forced motion of structures. J. Fluid Mech. 494, 141-162.

Newman, J. N. 1974 Interaction of water waves with two closely spaced vertical obstacles. J. Fluid Mech. 66, 97-106.

Newman, J. N. 1977 The motions of a floating slender torus. J. Fluid Mech. 83, 721-735.

Newman, J. N. 1999 Radiation and diffraction analysis of the McIver toroid. J. Engng Maths 35, 135-147.

UrSell, F. 1964 The decay of the free motion of a floating body. J. Fluid Mech. 19, 305-319. 